\title{
Gebelerde 50 gr Oral Glukoz Tolerans Test Sonuçları ile Vücut Kitle İndeksinin Karşılaştırılması
}

\author{
The Comparison of the $50 \mathrm{gr}$ Oral Glucose Tolerance Test Results with the Body Mass Index \\ in the Pregnancy Period of Women
}

\section{Selim KONCAGÜL ${ }^{1}$, Uğurkan ERKAYIRAN ${ }^{2}$, Zahide KÜÇÜK ${ }^{3}$}

1 Uzman Dr. Aksaray Eğitim ve Araştırma Hastanesi Kadın Hastalıkları ve Doğum Kliniği, AKSARAY

${ }^{2}$ Uzman Dr. Devakent Hastanesi Kadın Hastalıkları ve Doğum Kliniği ,KAHRAMANMARAŞ

${ }^{3}$ Uzman Dr. Etlik Lokman Hekim Hastanesi Kadın Hastalıkları ve Doğum Kliniği, ANKARAŞ

\section{Özet}

Amaç: Vücut kitle indeksi ile Gestasyonel Diyabetes Mellitus arasında doğrudan ilişki olduğu yapılan çalışmalarda gösterilmiştir. Bizim bu çalışmamızda amacımız 24-28 hafta gebeliği olan olgularımızın vücut kitle indeksleri ile 50 gr oral glukoz tolerans test sonuçlarını karşılaştırmak ve vücut kitle indeksine göre zayıf, normal kilolu, fazla kilolu, obez ve morbid obez olarak sınıflandırdığımız gruplar arasındaki oral glukoz tolerans test sonuçlarını değerlendirmektir.

Gereç ve Yöntem: Bu çalışmaya 2012 Kasım ayı ile 2013 Nisan ayları arasında polikliniğe başvuran 24-28 hafta gebeliği olan 200 hasta alındı. Hastalarımızın boy ve kilo ölçümleri yapıldı. Vücut Kitle İndeksleri hesaplanarak zayıf, normal, fazla kilolu, obez ve morbid obez olarak gruplara ayrıldı. $50 \mathrm{gr}$ glukoz oral yoldan günün herhangi bir saatinde hastanın açlığı gözetilmeden uygulandı. Vücut kitle indeksi ile 50 gr oral glukoz tolerans test sonuçları karşılaştırıldı.

Bulgular: Oral glukoz tolerans test sonrası glukoz değeri $140 \mathrm{mg} /$ dl ve üzerinde olan grupta, glukoz değeri $140 \mathrm{mg}$ /dl'den düşük olan gruba göre, vücut kitle indeksi $30 \mathrm{~kg} / \mathrm{m} 2$ ve daha yüksek olanların oranı istatistiksel olarak daha yüksek bulundu(p=0,048). Vücut kitle indeksi düzeyinin $25 \mathrm{~kg} / \mathrm{m} 2$ ' den düşük olanların oranı, 50 gr oral glukoz tolerans test sonucunda glukoz değeri $140 \mathrm{mg} / \mathrm{dl}$ 'den düşük olan grupta, oral glukoz tolerans testte ki glukoz değeri $140 \mathrm{mg} / \mathrm{dl}$ ve üzerinde olan gruba göre istatistiksel olarak anlamlı yüksek bulun$\mathrm{du}(\mathrm{p}=0,015)$. oral glukoz tolerans testteki glukoz değeri $140 \mathrm{mg} / \mathrm{dl}$ 'den düşük olan grup ile glukoz değeri $140 \mathrm{mg} / \mathrm{dl}$ ve üzerinde olan grup arasında, vücut kitle indeksi 25-29,9 arasında olanların oranı istatistiksel olarak benzer bulundu( $\mathrm{p}=0,581)$.

Sonuç: Obezite, Gestasyonel Diyabetes Mellitus için ciddi bir risk faktörüdür. Vücut kitle indeksi $30 \mathrm{~kg} / \mathrm{m} 2$ ve üstünde olan gebeler ilk antepartum vizitte diyabet açısından taranmalı eğer herhangi bir patolojik duruma rastlanılmazsa, 50 gr oral glukoz tolerans test 24-28. gebelik haftalarında tekrarlanılmalıdır. Bu hastaların antenatal kontrolleri daha sık yapılmalı ve mümkünse endokrinolog, diyetisyen ve kadın doğum uzmanından oluşan sabit bir ekipçe sürdürülmelidir.

Anahtar Kelimeler: Vücut kitle indeksi. OGTT 50 gr, Gestasyonel diabetes mellitus

\section{GİRIŞ}

Diyabet, gebelikte en s1k görülen medikal komplikasyondur (1). Gebelik, ikinci trimesterde başlayan insülin rezistansı, kompansatuar $\beta$-hücre

\section{Abstract}

Objective: The direct corellation between body mass index and gestational diabetes mellitus has been shown in recent studies. In this study, our aim is to compare our cases' body mass index with the results of $50 \mathrm{gr}$ oral glucose tolerance test who have 24-28 weeks gestation and to evaluate the oral glucose tolerance test results of the groups which are categorized as weak, normal weight, overweight, obese and morbidly obese according to body mass index.

Material and Methods: There were included 200 patients who referred to our hospital as having a 24-28 weeks gestation between the dates of November 2012 and April 2013. Our patients' height and weight measuements were performed. By calculating body mass index, they were grouped as weak, normal weight, overweight, obese and morbidly obese 50 gr of glucose were applied orally at any time of the day without considering patient's hunger.Body mass index and Oral Glucose Tolerance Test results were examined by comparing.

Results: Considering the group which had lower oral glucose tolerance test value than $140 \mathrm{mg} / \mathrm{dl}$, the rate of patients who have $30 \mathrm{~kg} /$ $\mathrm{m} 2$ and higher body mass index level was statistically higher in the group which has $140 \mathrm{mg} / \mathrm{dl}$ and higher(p=0.048). In the group of patients who had lower body mass index value than $25 \mathrm{~kg} / \mathrm{m} 2$, it was seen that the rate of patients who have lower oral glucose tolerance test value than $140 \mathrm{mg} / \mathrm{dl}$ was statistically much more than others. Between the group of patients who have lower oral glucose tolerance test value than $140 \mathrm{mg} / \mathrm{dl}$ and the group of patients who have $140 \mathrm{mg} / \mathrm{dl}$ and higher, the rate of patients who have body mass index value between 25 and 25.9 was found statistically similar( $\mathrm{p}=0.581)$.

Conclusion: Obesity is a serious risk factor for gestational diabetes mellitus. Pregnant women who have $30 \mathrm{~kg} / \mathrm{m} 2$ and higher body mass index value should be tested for diabets at the first antepartum visit and if there is not encountered any pathological situation $50 \mathrm{gr}$ oral glucose tolerance test should be repeated in 24-28 weeks gestation. These patients' antenatal checks should be performed more frequently and ,if possible, it should be continued with an unchanging team which includes an endocrinologist, dietician and an obstetrician.

Key Words: Body mass index, oral glucose tolerance test $50 \mathrm{mg}$, gestational diabetes mellitus

İletişim: $\begin{aligned} & \text { Uğurkan Erkayıran, Kahramanmaraş Devakent Hasta- } \\ & \text { nesi, Dulkadiroğlu/Kahramanmaraş }\end{aligned}$
DOI:
$\quad \mathbf{1 0 . 1 7 5 1 7 / k s u t f d . 3 0 2 6 2 3}$

artışı ve hiperinsülinemi ile karakterize diyabetojenik bir dönemdir. Gebelikte insülin sensitivitesi \% 80 oranında azalır (2). Gestasyonel diyabetes

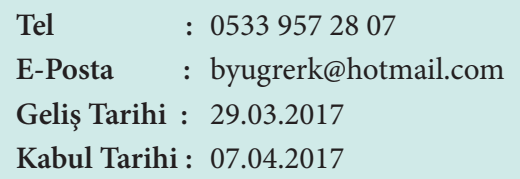


mellitus (GDM) ilk olarak gebelikte ortaya çıan ya da gebelik sirasinda tanı konulan glukoz tolerans bozukluğudur(3). GDM gebelerin yaklașık \%4'ünü komplike ederken, pregestasyonel diyabetin görülme sıklığ1 yaklaşık 1000 gebelikte 1-3'tür. Amerikan Diyabet Derneği gebe kadınların \%4'ünde GDM tespit edildiğini bildirmiştir (4). Fakat bu oran farklı toplumlarda \% 1 ile \% 14 arasında değişmektedir. Türkiye'de değişik yörelerde yapılan çalışmalarda, GDM prevalansının \% $3-8$ arasında değiştiği saptanmiştır (5).

GDM görülme sıklığını etkileyen önemli bir faktör anne yaşıdır. Yapılan çalışmalarda 25 yaş altı kadınlarda insidans \% 0,4-0,8 iken, 25 yaş üstü grupta bu oran \% 4,3-5,5 olarak bulunmuştur (6). Diğer risk faktörleri ise vücut kitle indeksinin(VKI) $\geq 27 \mathrm{~kg} / \mathrm{m}^{2}$ olmas1, etnik köken (siyah 1rkta daha fazla), birinci derece akrabalarında diyabet öyküsünün olması ve daha önceki gebeliğinde GDM öyküsü olmasıdır (7).

GDM'nin tanı ve taramasinın nasıl yapılması gerektiği halen netlik kazanmamıştır. Yalnız riskli gebelerin taranması ile GDM olgularının sadece \%50'si tanınabilmektedir. Günümüzde tarama testi olarak 50 gr ve tanı testi olarak 100 gr oral glukoz tolerans testi kullanılmaktadır. Dünya Sağlık Ổrgütü (DSÖ) 2 saatlik 75 gr oral glukoz tolerans testini önermekte ve bu uygulama bazı Avrupa ülkelerinde kabul görmektedir. Amerika Birleşik Devletleri'nde ise iki aşamalı test tercih edilmektedir $(8,9)$.

GDM plasental hormonların annedeki glukoz metabolizması üzerine etkileri sonucu genellikle 24. gebelik haftadan sonra ortaya çıkar. $\mathrm{Bu}$ hastalığın tanısını ve tedavisini atlamak maternal, gestasyonel, fetal ve neonatal komplikasyonlara sebep olabilir. GDM hipoglisemi, hiperglisemi, asidoz, retinopati, nefropati, nöropati gibi maternal komplikasyonlara; preeklampsi, polihidroamnioz, üriner enfeksiyon, preterm doğum, operatif doğum ve pelvik taban yaralanması gibi gestasyonel komplikasyonlara; abortus, konjenital anomaliler, makrozomi, fetal gelişme geriliği, perinatal mortalite gibi fetal komplikasyonlara; RDS(Respiratuar distres sendromu), hipoglisemi, hipokalsemi, hipomagnezemi, kardiomyopati gibi neonatal komplikasyonlara sebep olur (10).

Bizim bu çalışmada ki amacımız 24-28 hafta gebeliği olan hastaların VKİ ile 50 gr oral glukoz tolerans testindeki (OGTT) glukoz sonuçlarını karşılaştırmak ve VKİ'ne göre zayıf, normal kilolu, fazla kilolu, obez ve morbid obez olarak sınıflandırdığımız gruplar arasındaki OGTT sonuçlarını değerlendirmektir.

\section{GEREÇ VE YÖNTEMLER}

Çalışmamıza 2012 Kasım ayı ile 2013 Nisan ay1 arasında polikliniğe başvuran 24-28 hafta gebeliği olan 200 hasta alındı. Çalışma Helsinki Deklerasyonu 2008 prensiplerine uygun olarak yapıldı. Çalışma için Etik Kuruldan onay alındı. Hastalarımızın boy ve kilo ölçümleri yapılarak VKİ hesapland1. 50 gram glukoz oral yoldan günün herhangi bir saatinde hastanın açlığı gözetilmeden verildi. Alınan kan örneği Biyokimya Laboratuarına gönderildi. Glukoz verildikten bir saat sonra glukoz oksidaz yöntemi ile plazma glukozuna bakıldı. American Diabetes Association (ADA) ve American College of Obstetricians and Gynecologists (ACOG) plazmada eşik değer olarak $140 \mathrm{mg} / \mathrm{dL}$ 'yi önermektedir (11). Biz de çalışmamızda eşik değeri $140 \mathrm{mg} / \mathrm{dL}$ olarak kabul ettik.

Hastalarımızın VKİ değerleri boyun karesinin vücut ağırlığına oranı $(\mathrm{kg} / \mathrm{m} 2)$ formülünden hesaplandı. VKİ için DSÖ tarafından yapılmış olan siniflandırılma kullanıldı(12). Olgularımız 18,5 kg/ $\mathrm{m} 2$ altında ise zayıf, $18,5-24,9 \mathrm{~kg} / \mathrm{m} 2$ aras normal, $25-29,9 \mathrm{~kg} / \mathrm{m} 2$ aras1 fazla kilolu, 30- 39,9 kg/m2 aras1 obez ve $40 \mathrm{~kg} / \mathrm{m} 2$ üzerinde ise morbid obez olarak değerlendirildi (13).

$50 \mathrm{gr}$ OGTT $<139 \mathrm{mg} / \mathrm{dl}$ ise normal,

50 gr OGTT $140-200 \mathrm{mg} / \mathrm{dl}$ arası ise Bozulmuş OGTT,

50 gr OGTT $\geq 200 \mathrm{mg} / \mathrm{dl}$ ise GDM olarak kabul edildi (14).

\section{İstatistiksel Analiz}

Verilerin analizi SPSS for Windows 11.5 paket programında yapıldı. Tanımlayıcı istatistikler kategorik değişkenler ise gözlem sayısı ve (\%) biçiminde gösterildi. Glukoz yükleme testi sonuçlarına göre VKİ düzeylerinin dağılımında istatistiksel olarak anlamlı değişiklik olup olmadığ 1 Pearson'un Ki-Kare testi ile değerlendirildi. $p<0,05$ için sonuçlar istatistiksel olarak anlamlı kabul edildi.

\section{BULGULAR}

Hastaların \%64'ünün 50 gr OGTT deki kan glukoz değeri $140 \mathrm{mg} / \mathrm{dl}$ ' nin altında $\% 33,5$ 'inin $140-199 \mathrm{mg} / \mathrm{dl}$ arasinda, $\% 2,5$ 'inin ise $200 \mathrm{mg} /$ dl'nin üstünde olarak değerlendirildi.

Hastalarımız VKİne göre değerlendirildiklerinde \%5'inin $\mathrm{VKI}<18,5 \mathrm{~kg} / \mathrm{m}^{2}$, $\% 32,5$ 'inin VKI $18,5-24,9 \mathrm{~kg} / \mathrm{m}^{2}$ aras1, \%40,5'inin VKİ $25-29,9 \mathrm{~kg} / \mathrm{m}^{2}$ aras1, \%25'inin VKİ $30-39,9 \mathrm{~kg} /$ $\mathrm{m}^{2}$ aras $1, \% 1,5$ hastanın ise VKİ $40 \mathrm{~kg} / \mathrm{m}^{2}$ ve üstünde olarak değerlendirildi (Tablo 1).

50 gr OGTT sonucunda glukoz değeri 140 $\mathrm{mg} / \mathrm{dl}$ 'den düşük olan grupta VKİ düzeyinin 25 $\mathrm{kg} / \mathrm{m}^{2}$ ' den düşük olanların oranı, OGTT sonucu $140 \mathrm{mg} / \mathrm{dl}$ ve üzerinde olan gruba göre istatistiksel olarak daha yüksek bulundu( $\mathrm{p}=0,015)$. OGTT sonucu $140 \mathrm{mg} / \mathrm{dl}$ 'den düşük olan grup ile OGTT sonucu $140 \mathrm{mg} / \mathrm{dl}$ ve üzerinde olan grup arasinda VKI 25-29,9 arasinda olanların oranı istatistiksel olarak benzer bulundu( $\mathrm{p}=0,581)$. VKİ $30 \mathrm{~kg} / \mathrm{m}^{2}$ 'nin üzerinde olan hastalar glukoz değerlerine göre kıyaslandığında glukoz değeri $140 \mathrm{mg} / \mathrm{dl}$ ve üzerinde olanların oranı glukoz değeri $140 \mathrm{mg} / \mathrm{dl}$ 'nin altında olanlara göre istatistiksel olarak anlamlı yüksek değerlendirildi $(p=0,048)$ (Tablo 2).

\section{TARTIŞMA}


Tablo 1: OGTT Düzeylerine Göre VKİ Yönünden Olguların Frekans Dă̆ılımı

\begin{tabular}{|l|c|c|c|c|}
\hline & $<140 \mathrm{mg} / \mathrm{dl}$ & $140-200 \mathrm{mg} / \mathrm{dl}$ & $\geq 200 \mathrm{mg} / \mathrm{dl}$ & Toplam \\
\hline$<18,5 \mathrm{~kg} / \mathrm{m}^{2}$ & $1(\% 0,8)$ & - & - & $1(\% 0,5)$ \\
\hline $18,5-24,9 \mathrm{~kg} / \mathrm{m}^{2}$ & $49(\% 38,3)$ & $15(\% 22,4)$ & $1(\% 20,0)$ & $65(\% 32,5)$ \\
\hline $25-29,9 \mathrm{~kg} / \mathrm{m}^{2}$ & $50(\% 39,1)$ & $28(\% 41,8)$ & $3(\% 60,0)$ & $81(\% 40,5)$ \\
\hline $30-39,9 \mathrm{~kg} / \mathrm{m}^{2}$ & $26(\% 20,3)$ & $23(\% 34,3)$ & $1(\% 20,0)$ & $50(\% 25,0)$ \\
\hline$\geq 40 \mathrm{~kg} / \mathrm{m}^{2}$ & $2(\% 1,6)$ & $1(\% 1,5)$ & - & $3(\% 1,5)$ \\
\hline Toplam & $128(\% 64,0)$ & $67(\% 33,5)$ & $5(\% 2,5)$ & $200(\% 100,0)$ \\
\hline
\end{tabular}

Dünyada obezite prevelansinin (VKI $>30$ $\left.\mathrm{kg} / \mathrm{m}^{2}\right) \% 15-20$ olduğu, 15 ve üzeri yaș grubunda ve üreme yaşındaki kadınlarda obezite riskinin giderek $\operatorname{artt1ğ} \quad$ belirtilmektedir (17). Amerika da yapilan bir araștırmada, 2003-2006 y1lları arasinda ise 2044 yaş aralığındaki obez kadın oranının \% 32'ye yükseldiği belirtilmiştir (18). Birçok ülkede gebelik öncesinde obezite ve fazla

Diyabet ile komplike olmuş gebelikler, hem maternal hem fetal açıdan dikkatli takip gerektiren riskli gebeliklerdir. Yeterli glisemik kontrol sağlanamadığında, bebekte konjenital malformasyondan in utero ölüme, annede hipoglisemi, diyabetik ketoasidoz, retinopati, nöropati ve nefropatide artışa neden olarak morbidite ve mortalitede artışa neden olabilen metabolik bir bozukluktur. Bu komplikasyonların iyi bir glisemik kontrolle önlenebilir olması ise yüz güldürücü yanıdır. İlk defa 1922'de insülinin keşfiyle yeni bir dönem başlamış, maternal mortalite \% 30'lardan günümüzde $\% 0$ 'lara, perinatal mortalite ise $\%$ 60'lardan \% 5 değerlerine düşmüştür (10). 50 gr OGTT tarama amaciyla kullanılan bir testtir. $\mathrm{Bu}$ testin sonucunda glukoz değeri $140 \mathrm{mg} / \mathrm{dL}$ ve üzerindeyse gestasyonel diyabeti olanların $\%$ 80'i tanınabilmektedir. Bu değer $130 \mathrm{mg} / \mathrm{dl}$ olarak alınırsa gestasyonel diyabeti olanların \% 90'1 tanınabilir fakat bu grupta \% 20-25 oranında yalancı pozitiflik gözlenir, eşik değer $140 \mathrm{mg} / \mathrm{dl}$ olursa yalanc1 pozitiflik oran1 \% 14-18 olacaktır. Yapılan iki çalıșma göstermiștir ki GDM olan hastaların \% 10'unun tarama değerleri 130-139 $\mathrm{mg} / \mathrm{dL}$ arasındadır $(7,15)$. Eşik değer $130 \mathrm{mg} / \mathrm{dL}$ alındığında sensitivite \% 10 artacak fakat tanısal glukoz tolerans testinin uygulanması popülasyonun $\% 14$ 'ünden \%23'e çıkarak maliyeti artıracaktır (15). ADA ve ACOG plazmada eșik değer olarak $140 \mathrm{mg}$ / dL'yi önermektedir (16). Biz de çalışmamızda eşik değeri $140 \mathrm{mg} / \mathrm{dL}$ olarak kabul ettik.

Tablo 2: OGTT Düzeylerine Göre VKİ Yönünden Olguların Frekans Dağılımı

\begin{tabular}{|l|l|l|l|}
\hline & $<140 \mathrm{mg} / \mathrm{dl}(\mathrm{n}: 128)$ & $\geq 140 \mathrm{mg} / \mathrm{dl}$ (n:72) & p-değeri \\
\hline$<25 \mathrm{~kg} / \mathrm{m}^{2}$ & $50(\% 39,1)$ & $16(\% 22,2)$ & 0,015 \\
\hline $25-29,9 \mathrm{~kg} / \mathrm{m}^{2}$ & $50(\% 39,1)$ & $31(\% 43,1)$ & 0,581 \\
\hline$\geq 30 \mathrm{~kg} / \mathrm{m}^{2}$ & $28(\% 21,8)$ & $25(\% 34,7)$ & 0,048 \\
\hline
\end{tabular}
değerlendirildi. Kilolu olma prevalansını̈ gebe kadınlar arasında arttığı görülmektedir. DSÖ kriterlerine göre dünyada gebe kadınlardaki obezite prevalansı (VKI>30 kg/ $\mathrm{m}^{2}$ ) \%1,8 ile \%25,3 arasında değişmektedir (19). 2006 yılı verilerine göre İngiltere' de gebe kadınlarda ki obezite prevalansı \%18,5; Amerika'da ise bu oranın \% 18,5 ile \% 38,3 olduğu belirtilmektedir (20). Ülkemizde yapılan bir araştırmada ise gebe kadınların \% 27,2'sinin fazla kilolu ve obez olduğu belirlenmiştir (21). Bizim çalışmamızda ise hastalarımızın \%25'inin (n:50) VKİ 30-39.9kg/ $\mathrm{m}^{2}$ arasinda olup fazla kilolu, \%1,5 hastanın (n:3) ise VKI $40 \mathrm{~kg} / \mathrm{m}^{2}$ ve üzerinde olup obez olarak

VKI'nin artması gebelikte GDM gelișme ihtimalini artırmaktadır (12). GDM'nin maternal fetal komplikasyonları artırdığı ve obezitenin ve aşırı kilo alımının GDM'ye ait bu komplikasyonların oluşma ihtimalini artırdığ gösterilmiştir (19). Yapılan birçok çalışma VKI’nin artmasının GDM'nin gebelikteki komplikasyonları artırdığını göstermiştir (22, 23). Az sayıdaki bazı çalışmalarda ise VKİ yüksek olanlarda glukoz toleransının değişmediğini ve GDM olasılığını artırmadığını gözlemişlerdir (24-26). Obez olan hastaların kilo vermesi halinde nondiyabetikler gibi veya normal popülasyondaki gebeler gibi GDM riskinin değişmediği gösterilmiştir(27,28). Bizim çalışmamızdan çıkan sonuçları incelendiğimizde VKI arttıkça 50 gram OGTT’deki glukoz değerinin arttığını gördük. OGTT deki glukoz sonucu 140 $\mathrm{mg} / \mathrm{dl}$ ve üzerinde olan grupta, OGTT'deki glukoz sonucu $140 \mathrm{mg} /$ dl'den düşük olan gruba göre VKI'ne göre fazla kilolu ve obez olanların oranı istatistiksel olarak daha yüksekti $(p=0,048)$. Bununla birlikte OGTT deki glukoz değeri 140 mg/dl'den düşük olan grupta VKI düzeyinin $25 \mathrm{~kg} / \mathrm{m}^{2}$ den düşük olanların oranı OGTT değeri $140 \mathrm{mg} / \mathrm{dl}$ ve KSÜ Tip Fak Der 2017;12(1)45-49 
üzerinde olan gruba göre istatistiksel olarak daha yüksekti $(p=0,015)$. OGTT sonucu $140 \mathrm{mg} / \mathrm{dl}$ 'den düşük olan grup ile OGTT sonucu $140 \mathrm{mg} / \mathrm{dl}$ ve üzerinde olan grup arasinda VKİ 25-29,9 arasinda olanların oranı istatistiksel olarak benzer bulundu $(p=0,581)$.

Sonuç olarak, obezite GDM için ciddi bir risk faktörüdür. VKİ arttıkça GDM ve değişik derecede glukoz intoleransı açığa çıkma riski artar. VKİ 30 $\mathrm{kg} / \mathrm{m}^{2}$ ve üstünde olan gebeler ilk antepartum vizitte diyabet açısından taranmalı eğer herhangi patolojik bir duruma rastlanılmazsa, 50 gram OGTT 24-28. haftalarda tekrarlanılmalıdır. Bu hastaların antenatal kontrolleri daha sık yapılmalı ve mümkünse endokrinolog, diyetisyen ve kadın doğum uzmanından oluşan sabit bir ekipçe sürdürülmelidir.

Yazarlar arasında herhangi bir çıkar çatışması bulunmamaktadır. Çalışma için herhangi bir finansal destek yoktur.

\section{KAYNAKLAR}

1. Nedim Çiçek M, Akyurek C, Çelik C, Haberal A. Diabetes mellitus ve gebelik. Kadın Hastalıkları ve Dogum Bilgisi 2006: 435-450.

2. Carpenter MW, Coustan DR. Criteria for screening tests for gestational diabetes mellitus. Am Obstet Gynecol 1982; 144: 768.

3. Turok DK, Ratcliffe SD, Baxley AG. Management of gestational diabetes mellittus. Am Fam Physician 2003; 68: 1769-1772.

4. Landon MB, Spong CY, Thom E, Carpenter MW, Ramin SM, Casey B, et al, Eunice Kennedy Shriver National Institute of Child Health and Human Development Maternal-Fetal Medicine Units Network. A multicenter, randomized trial of treatment for mild gestational diabetes. $\mathrm{N}$ Engl J Med 2009; 361:1339-1348.

5. Özçimen EE, Uçkuyu A, Çiftçi FC, Yanık FF, Bakar C. Diagnosis of gestational diabetes mellitus by use of the homeostasis model assessment insulin resistance index in the first trimester. Gynecol Endocrinol 2008: 24: 224249.

6. Marquette GP, Klein VR, Niebyl JR. Efficacy of screening for gestational diabetes. Am J Perinatol 1985:2: 7-9.

7. Gestational diabetes. American Diabetes Association. http://www.diabetes.org/diabetesbasics/gestational/what-is-gestational-diabetes. html. Accessed Dec. 10, 2013.

8. Hills S. DIAMAP - mapping the future of diabetes research. Diabetes Voice. 2009;54: 458.

9. Halban PA. Prime time for DIAMAP: A road map for diabetes research in Europe. Diabetologia. 2010; 53: 1835-7.

10. Kuhl C. Glucose metabolism during and after pregnancy in normal and gestational diabetic woman. Acta Endocrinol 1995;79: 709-719.

11. ACOG Technical bulletin: Diabetes and pregnancy. Number 200, December 1994. Int J Gynecol Obstet 1995,48:331-339.

12. Abenhaim HA, Kinch RA, Morin L, Benjamin A, Usher R. Effect of prepregnancy body mass index categories on obstetrical and neonatal outcomes. Arch Gynecol Obstet 2007; 275: 3943.

13. World Health Organization W. Obesity and overweight. http://www.who.int/mediacentre/ factsheets/ fs311/en/: 2016 June 2016.

14. American Diabetes Association. Diagnosis and classification of diabetes mellitus. Diabetes Care 2014;37 (Suppl. 1): S81-S90.

15. Couston D. Maternal age and screening for ges-tational diabetes: A population based study. Obstet Gynecol 1989;73: 557-561.

16. American College of Obstetricians and Gynecologists: Diabetes and pregnancy. ACOG Technical Bulletin. Washington, DC 1994.

17. Stodhard KJ, Tennent PWG, Bell R, Rankin J. Maternal overweight and obesity and risk of congenital anomalies. A systemic rewiev and meta- analysis. JAMA. 2009;301: 636-50.

18. Drake AJ, Reynolds RM. Impact of maternal obesity on offspiring obesity and cardiometabolic disease risk. Reproduction. 2010:140; 387-398.

19. Yee LM, Cheng YW, Inturrisi M, Caughey AB. Effect of gestational weight gain on perinatal outcomes in women with type 2 diabetes mellitus using the 2009 Institute of Medicine guidelines. American Journal of Obstetrics and Gynecology 2011; 205, 257.e251-256.

20. Guelinckx I, Devlieger R, Beckers K, Vansant G. Maternal obesity: pregnancy complications, gestational weight gain and nutrition. Obes Rev. 2008; 9:140-50

21. 21. Daşıkan Z, Kavak O. Maternal obesity: Pregnancy complications an managemant of pregnant woman: Review [Maternal obezite: gebelik komplikasyonları ve gebe kadın yöntemi]. Türkiye Klinikleri J NUrs Sci. 2009;1: $39-46$.

22. Metzger, B. E. et al. International association of diabetes and pregnancy study groups recommendations on the diagnosis and classification of hyperglycemia in pregnancy. Diabetes Care 2010; 33. 676-682.

23. Dodd JM, Grivell RM, Nguyen AM, Chan A, Robinson JS. Maternal and perinatal health outcomes by body mass index category. The Australian and New Zealand Journal of Obstetrics \& Gynaecology 2011; 51: 136-140.

24. Owens LA, O'Sullivan EP, Kirwan B, Avalos 
G, Gaffney G, Dunne F; ATLANTIC DIP Collaborators. ATLANTIC DIP: the impact of obesity on pregnancy outcome in glucosetolerant women. Diabetes Care 2010; 33: 577 579 .

25. Catalano PM, McIntyre HD, Cruickshank JK, McCance DR, Dyer AR, Metzger BE, et al. The hyperglycemia and adverse pregnancy outcome study: associations of GDM and obesity with pregnancy outcomes. Diabetes Care 2012; 35.

26. Dennedy MC, Avalos G, O'Reilly MW, O'Sullivan EP, Gaffney G, Dunne F. ATLANTIC-DIP: raised maternal body mass index (BMI) adversely affects maternal and fetal outcomes in glucose-tolerant women according to International Association of Diabetes and Pregnancy Study Groups (IADPSG) criteria. Journal Clin Endocrinol Metab 2012; 97: E608E612.
27. Nohr EA, Vaeth M, Baker JL, Sørensen TIa, Olsen J, Rasmussen KM. Combined associations of prepregnancy body mass index and gestational weight gain with the outcome of pregnancy. Am J Clin Nutr 2008; 87: 1750-1759.

28. Black MH, Sacks DA, Xiang AH, Lawrence JM. The relative contribution of prepregnancy overweight and obesity, gestational weight gain, and IADPSG-defined gestational diabetes mellitus to fetal overgrowth. Diabetes Care 2013; 36: 56-62. 